C2006 IEEE. Personal use of this material is permitted. However, permission to reprint/republish this material for advertising or promotional purposes or for creating new collective works for resale or redistribution to servers or lists, or to reuse any copyrighted component of this work in other works must be obtained from the IEEE. 


\title{
Defining Trustworthiness in Service Oriented Environment
}

\author{
Farookh Khadeer Hussain ${ }^{1}$, Elizabeth Chang $^{1}$ and Tharam S.Dillon ${ }^{2}$ \\ ${ }^{1}$ School of Information Systems \\ Curtin University of Technology \\ P.O. Box U1987 \\ Perth, Western Australia, Australia \\ \{Farookh.Hussain, Elizabeth.Chang\}@cbs.curtin.edu.au \\ ${ }^{2}$ Faculty of Information Technology \\ University of Technology, Sydney \\ Sydney, NSW 2007, Australia \\ tharam@it.uts.edu.au
}

\begin{abstract}
In the existing literature we note that there has been no effort in proposing a definition of trustworthiness. In this paper, we propose a definition of trustworthiness with focus on service oriented environments. In addition, we propose and discuss in detail the various factors which can affect the trustworthiness assigned by the trusting agent to the trusted agent.
\end{abstract}

\section{Introduction}

After an interaction, the trusting agent (or product or service) has to assign a value which would correspond to the trust the trusting agent has in the trusted agent or product or service. This value assigned by the trusting agent to the trusted agent/ product or service becomes the reputation of the trusted agent (or product or service) when the trusting agent communicates it to the other agents when they ask it for recommendations. Hence we can see that assigning a trust value to the interacting counterpart is essential because of the following reasons

- The assigned trust value denotes the Quality of the Trusted Agent, or the Quality of Service or the Quality of Product.

- Other agents who have not interacted with the given trusted agent could make use of the assigned trust value by the trusting agent in order to make a trust based decision of whether or not they should do ahead and interact with the trusting agent or service or product.

Unfortunately there is little work in the area of how the trusting agent could assign a trust value to the trusted agent. In this paper we propose the concept of trustworthiness as means which the trust that the trusting agent has in the trusted agent or product or service can be quantified, expressed and subsequently communicated to the other reputation querying agents.

This paper is organized as follows,

In Section 2, we propose a definition of trustworthiness. In Section 3, we propose the characteristics of trustworthiness. Section 4 concludes the paper. Due to space constraints we are unable to discuss the contents of the paper in detail. We encourage interested readers to refer to [1] on a thorough discussion and analysis on trustworthiness.

\section{Trustworthiness}

In this section we define what we mean by trustworthiness and explain in detail what the definition of trustworthiness.

Trustworthiness is defined as a numeric value that depicts the level of trust between in a trust relationship.

In the following sections, we explain the above concepts in detail.

\section{1 "a numeric value" as defined for} Trustworthiness

By the term "a numeric value" we mean a ranking or rating that quantifies the amount of trust between the trusting agent and the trusted agent. This ranking or rating can be either a non-numeric rating or a numeric rating although we later suggest the use of an integer.

We define the numeric rating of a trust relationship as an assessment of a trust relationship expressed in terms of an integer or real numbers. 
We define the non-numeric rating of a trust relationship as a valuation of a trust relationship expressed neither in terms of an integer nor in terms of real numbers.

Rahman [2] had proposed four different levels to quantify the amount of trust between two agents ('Very Trustworthy', 'Trustworthy', 'Untrustworthy', 'Very Untrustworthy'). The ratings proposed by Rahman [2] are an example of non-numeric rating. Cornelli and Damiani [3] proposed a non-numeric rating in terms of a number of '*', with each additional '*' denoting a higher level rating.

However Rahman [2] and Cornelli and Damiani [3] do not define the semantics associated with the various trustworthiness levels. They do not formally define under what conditions an agent can be regarded as "Very Untrustworthy' or 'Trustworthy'. Nor do Cornelli and Damiani [2] present a formal definition of trustworthiness.

Aberer [4], Xiong [5], Chen [6], Kamvar [7], Wang [8] all propose the use of a numeric value to quantify the amount of trust between two agents. However their approaches also suffer from the shortcomings that we mentioned for Rahman [2] and Cornelli and Damiani [3].

Another dimension along which trustworthiness of an entity can be classified is whether a discrete value or a global value is used to quantify the amount of trust between the agents.

Global trustworthiness value of an agent as a universal numeric rating or non-numeric rating that is associated with an agent and reflects the extent to which an agent can be regarded as trustworthy. In the global trustworthiness value of an agent, each agent is associated with a single trustworthiness value that captures the extent to which it can be regarded as trustworthy. The trustworthiness value of an agent after each rating is added to the previous global value. This single global trustworthiness value associated with an agent captures and reflects its behaviour in the past.

We define a discrete trustworthiness value of an agent as a numeric rating or a non-mumeric rating that is associated with an agent and reflects the extent to which an agent can be regarded as trustworthy. In contrast to the global trustworthiness value of an agent, there is no global value. Each trustworthiness value after an interaction with an agent is stored and treated separately from the previous trustworthiness values.

Chen [6], propose 5 different trustworthiness levels $[-1,4]$ and makes use of discrete trustworthiness levels. Wang [8] propose a trustworthiness domain of $[0,1]$ and also makes use of discrete trustworthiness levels. However, Wang [8] does not propose different trustworthiness levels in the range of [ 0,1]. Rahman [2] and Cornelli and Damiani [3] also propose the use of discrete trustworthiness values.

Aberer [4], Xiong [5], Kamvar [7] propose the use of a global trustworthiness value for an agent. They do not propose different trustworthiness levels in their trust model.

\section{2 "level of trust" as defined for Trustworthiness}

The term "level of trust" means that rating or ranking quantifies the amount of trust between the trusting agent and the trusted agent. In other words trustworthiness is a measure to express the amount or quantity of trust between that the trusting agent has in the trusted agent.

As discussed in our previous publications [1], trust between a trusting agent and a trusted agent is realised by trust relationship. The amount or the level of trust between the trusting agent and the trusted agent, involved in a trust relationship can be deduced by a comparison of the intensity of the trust relationship between them and the maximum possible intensity that can be assigned to any trust relationship. In other words trustworthiness communicates the intensity of the trust relationship but it does so relative to the highest available trustworthiness value.

As an example let us assume that Alice is the trusting agent and Bob is the trusted agent. Further more let us assume that Alice assigned a trustworthiness value of ' 6 ' to Bob. This value ' 6 ' of the strength or intensity of the trust relationship between Alice and Bob does not convey any information at all. But if we assume that the maximum possible trustworthiness value of any trust relationship is ' 6 ' then we can surmise that Alice considers Bob to be very trustworthy indeed.

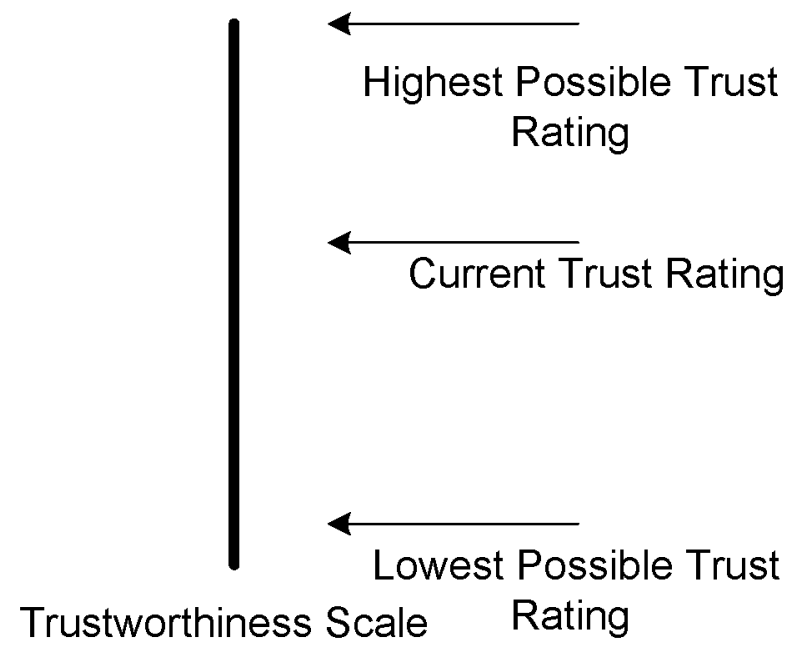

Figure 1: Trustworthiness Values are expressed relative to the highest possible trustworthiness value 
On the contrary, if we assume that the maximum possible trustworthiness value for any trust relationship is ' 10 ' then we can surmise that Alice consider Bob to be worthy of average trustworthiness (or of a trustworthiness level which does not correspond to the maximum trustworthiness).

In other words the numeric trustworthiness value reflects the strength of the trust relationship. This reflection of the strength of the trust relationships is derived by a comparison between the highest possible trustworthiness value of any relationship and the trustworthiness of that particular trust relationship has. This is an important feature of trustworthiness

The semantics of the trusting agent and the trusted agent while defining trustworthiness is the same as the semantics of the trusting agent and the trusted agent while defining trust.

\section{3 "Trustworthiness"}

As shown in Figure 2, trust or belief between two agents is realized by a trust relationship between them. Each context in which the trusting agent trusts the trusted agent leads to a distinct trust relationship. Trustworthiness is a measure to quantify the strength or intensity of the trust relationship. This measure of the trust relationship is dependent on many factors which I will out line in Section 2. A given trustworthiness value for a given trustworthiness shows the intensity of a single trust relationship.

In other words trustworthiness denotes the strength or intensity of a single trust relationship. Each trust relationship should be quantified by exactly one numeric value denoting the intensity of the trust relationship.

The following diagram intends to capture the relationship between trust, trust relationship and trustworthiness and the properties of the trustworthiness.

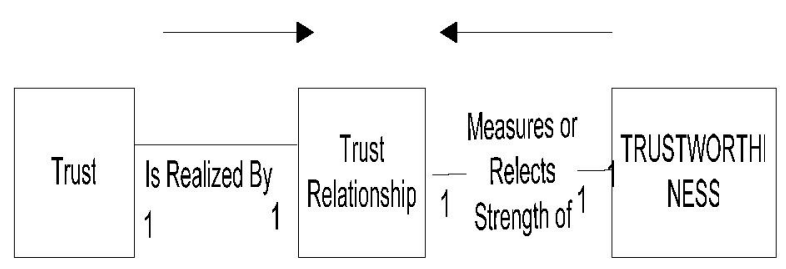

Figure 2: Conceptual Representation of the relationship between the concepts of Trust, Trust Relationship and Trustworthiness.

As can be seen from the above figure, trust by a trusting agent in a trusted agent, leads to the formation of a trust relationship between the trusting agent and trusted agent.
The trust relationship is unidirectional in a direction from the trusting agent to the trusted agent. Trustworthiness quantifies and expresses the strength of the trust relationship. Each belief that the trusting agent has in the trusted agent, leads to a unique trust relationship. Each trust relationship in turn is quantified and expressed by a single value.

\section{Properties of Trustworthiness}

In this section we will propose the properties of trustworthiness. The properties of trustworthiness are closely aligned with the properties of trust. The properties of trustworthiness are as below,

\subsection{Dynamic Nature of Trustworthiness}

As discussed in our earlier publication [1] that trust is dynamic. Trust is dynamic because the belief that the trusting agent has in the trusted agent or service or product may or may not be static. When the trust between a given trusting agent and trusted agent changes, the intensity of the trust relationship between them in order to reflect the change in the trust. If the trust that the trusting agent has in the trusted agent increases then the intensity of the trust relationship will increase and vice versa.

Since trustworthiness is a numeric value that communicates the intensity of the trust relationship, if the intensity of the trust relationship changes then the trustworthiness too has to change accordingly in order to reflect the change in the intensity of the trust relationship. The trustworthiness value has to change in order to manifest the change the strength of the trust relationship which in turn has to change in order to manifest the change in the trust between the trusting agent and the trusted agent.

Continuing with the above example of the trust relationship between Alice and Bob, let us assume that Alice upon further interaction/s with Bob found out that Bob is very untrustworthy. As a result of this, Alice will reduce the belief or trust that she has in Bob. As a result of this reduction in trust, the intensity of the trust relationship that Alice has with Bob will decease. As we pointed out the trustworthiness denotes the intensity of a trust relationship, trustworthiness too has to change to reflect the change in the intensity of the trust relationship.

\subsection{Context-Specific Nature of Trustworthiness}

As discussed in our earlier publication [1], trust is context specific, because of the following reasons:

- Trust in another agent is a belief or opinion about that agent. 
- This belief that the trusting agent has in a given scenario may or may not hold in some other scenario.

Additionally we mentioned before that if two or more agents trust each other they form or enter a trust relationship with each other. Thus, if trust is contextdependent, trust relationship between two agents is valid only in the situation in which the agents trust each other.

Since trustworthiness is a numeric value that denotes the intensity of the trust relationship between two agents, trustworthiness inherently is context dependent.

The intensity of the trust relationship between two agents may be; more, less or non-existent as compared to the intensity of the trust relationship in another scenario. Correspondingly the numeric rating denoting the strength of the trust relationship cannot be the same over all the different trust relationships.

Each trust relationship should be quantified by exactly one numeric value denoting the intensity of the trust relationship (put this in the end). This trustworthiness value should additionally be annotated by a time slot (due to space constraints it is difficult to explain in detail the notion of time slot. Interested readers are encouraged to refer to Chang et al [1] for an in-depth discussion on time slot). The trustworthiness value assigned by a trusting agent to the trusted agent in a given context and time slot may not be necessarily be applicable in another context and time slot.

Let us consider two agents, Alice and Bob. Let us assume two contexts, 'lending a car' and 'lending a house'. Let us assume that Alice trusts Bob to lend its house. However this does not imply that Alice will trust Bob with his car as well. It may be the case that Bob does not know how to drive and as a result Alice does not trust him with his car. The trust that Alice has in Bob in the context of 'lending his house' does not convey any thing about the trust that the Alice has in Bob in the context of 'lending his car' or any other context. All it conveys is the trust that Alice as the trusting entity has in the Bon as the trusted agent in the context of 'lending his house'. Hence the intensity of the trust relationship that Alice has with Bob in the scenario of lending his house may not necessarily be the same as the intensity of the trust relationship that Alice has with Bob in the context of lending his car or any other context.

Hence the rating denoting the intensity of the trust relationship in a given context may or may not be able to denote the intensity of the trust relationship in another context.

\section{3 "Intrinsic type of Agents" for Trustworthiness}

The rating denoting or quantifying the amount of trust is dependent upon the intrinsic quality of the agents involved in the trust relationship. The term "intrinsic type of agents" captures:

- The quality of the trusted agent.

- The psychological type of the trusting agent.

The rating assigned by the trusting agent to the relationship that it shares with the trusted agent is dependent on the quality of the trusted agent. If the trusted agent is of high quality then the relationship will be assigned a high rating by the trusting agent. On the contrary if the trusted agent is not of good quality then it will be assigned a low rating.

We make use of the term "quality of agent" as an abstraction of the willingness and capability of the agent to deliver on the mutually agreed behavior.

The "psychological type of trusting agent" takes into account the personal nature of trust as well. We pointed out in an earlier publication [1] that trust is personal and has a personal touch attached to it. The term "psychological type of trusting agent" captures the subjective nature and the personal nature associated with the intensity of the trust relationship.

Trusting Agents who have a 'Thinking' psychological preference will analyze things in an objective and logical fashion and assign trustworthiness values (in other words, decide the intensity of the trust relationship) to the trusted agent based on the factors that they have analyzed logically and in an objective fashion. In our opinion the agents with 'Thinking' preference, when deciding the intensity of the trust relationship (assigning trustworthiness value to the trusted agent), will not pay importance to the personal rapport that they share with the trusted agent.

On the contrary, Trusting Agents who have a 'Feeling' preference will not analyze things in an objective and logical fashion and put more weight to the personal rapport that they share with the trusted agent, when deciding the strength or intensity of the trust relationship with the trusted agent.

Let us consider an example to explain our argument.

Assume that East Field, West Field and South Field, are three logistic companies. Let us further assume that they are all located in distinct places and furthermore let us assume that West Field and South Field want to use the warehouse space of East Field.

After West Field has used the warehouse space of East Field, it will as the trusting agent assign a trustworthiness value to East Field as the trusted agent. Similarly, South Field after its interaction with East Field will assign a trustworthiness value to East Field. 
Let us assume that East Field provided the same quality of service to both East Field and West Field.

It is quite possible that for the same quality of service provided by East Field, West Field and South Field do not assign the same trustworthiness value to East Field. It is possible that for the same quality of service provided by East Field, West Field and South Field have two different perceptions of the quality of service and hence assign different trustworthiness values to East Field.

It is possible that South Field assigns a high trustworthiness value as it perceives the quality of service provided by East Field as good. On the contrary West Field might assign an average trustworthiness value as it perceives the quality of service provided by East Field as mediocre. Additionally it is possible that West Field has a personal affinity with East Field and it assigns a higher trustworthiness value to East Field.

These two different interpretations of the Same Quality of Service by West Field and South Field are the personal opinion and personal judgments of the Trusting Agents them selves leading to distinct trustworthiness values for East Field.

Additionally let us assume that both South Field and West Field have a personal rapport with East Field. Let us further assume that South Field has a 'Feeling' psychological preference while East Field has a 'Thinking' psychological preference. It is possible that South Field due to the personal rapport that it has with East Field assigns a high trustworthiness value to East Field (taking into account the personal rapport that it shares with East Field)

On the contrary, as East Field which has a 'Thinking' psychological preference analyses the behaviour of South Field in an objective way with no regard for the personal rapport that it has with East Field.

Hence the trustworthiness value assigned by West Field and South Field to East Field may or may not be the same, for the same Quality of Service provided by East Field.

These two different trustworthiness values assigned by West Field and South Field to East Field are a result of the different psychological nature of the Trusting Agents, West Field and South Field. As a result of these different psychological natures we get two different interpretations for the same quality of service and hence two different trustworthiness values.

Hence we feel that the rating assigned to the trust relationship that the trusting agent shares with the trusted agent is subjective and depends on the psychological trait of the Trusting Agent.

\section{Summary}

From an extensive review of the existing literature we note that there has been no effort in proposing a definition of trustworthiness. In this paper, we addressed this issue by proposing a definition of trustworthiness with focus on service oriented environments. Although this definition has been proposed from the perspective of service oriented environment, this definition of trustworthiness holds for any e-business scenario.

In addition, we have proposed and discussed in detail the various factors which can affect the trustworthiness assigned by the trusting agent to the trusted agent.

\section{Reference}

[1]Elizabeth Chang, Tharam Dillon, Farookh Khadeer Hussain (2006), Trust and Reputation for Service Oriented Environment, John Wiley and Sons, To appear in 2006.

[2]Alfarez Abdul-Rahman, Stephen Hailes 2000, Supporting Trust in Virtual Communities, Available:[http://citeseer.nj.nec.com/cache/papers/cs/10 496/http:zSzzSzwww-dept.cs.ucl.ac.ukzSzcgibinzSzstaffzSzF.AbdulRahmanzSzpapers.plzQzhicss33. pdf/abdul-rahman00supporting.pdf] (30/11/2003).

[3]Fabrizo Cornelli, Ernesto Damiani, Sabrina De Capitani di Vemarcati,Stefano Paraboschi,Pierangela Samarati 2003, Choosing Reputable Servants in a P2P Network, Available: [http://citeseer.nj.nec.com/cache/papers/cs/26951/http:zS zzSzseclab.crema.unimi.itzSzPaperszSzwww02.pdf/cho osing-reputable-servents-in.pdf] (10/10/2003).

[4]Karl Aberer, Zoran Despotovic, Managing Trust in a Peer-2-Peer Information System, Available: [http://citeseer.nj.nec.com/cache/papers/cs/26315/http:zS zzSzwww.p-

grid.orgzSzPaperszSzCIKM2001.pdf/aberer01managing .pdf] $(10 / 10 / 2003)$.

[5]Li Xiong, Ling Liu 2002, Building Trust in Decentralized Peer-to-Peer Electronic Communities, Available:

[http://citeseer.nj.nec.com/cache/papers/cs/26940/http:zS zzSzdisl.cc.gatech.eduzSzPeerTrustzSzpubzSzxiong02b uilding.pdf/xiong02building.pdf] (10/04/2003).

[6] Wang, Y., Vassileva J, Trust and Reputation Model in Peer-to-Peer, Available:

[www.cs.usask.ca/grads/yaw 181/

publications/120_wang_y.pdf] (15/10/2003). 
[7] Kamvar, Sepandar D., Schlosser, Mario T., GarciaMolina, Hector, The EigenRep Algorithm for Reputation Management in P2P Networks, Available:

[http://citeseer.nj.nec.com/kamvar03 eigentrust.html] $(11 / 9 / 2003)$.

[8]Wang, Y., Vassileva J., Bayesian Network Trust Model in Peer-to-Peer Networks, Available:

[http://bistrica.usask.ca/madmuc/Pubs/yao880.pdf] $(11 / 9 / 2003)$ 